\title{
An Iterative Multiuser Detection/Decoding for Nonbinary LDPC Coded MC-MFSK Multiple Access Systems
}

\author{
Szu-Lin Su, Her-Chang Tsai \\ Institute of Computer and Communication Engineering, National Cheng Kung University, Tainan, Taiwan \\ ssl@ee.ncku.edu.tw
}

\begin{abstract}
This paper studies an iterative multiuser detection and decoding for nonbinary low density parity check (LDPC) coded multicarrier M-ary frequency shift keying (MC MFSK) multiple access system to increase system spectral efficiency under specific signal-to-noise ratio. At multiuser detection side, soft metric for each symbol is quantized to 2 levels (2L) or 3 levels (3L) and then pass to the decoder. When the soft output of a symbol from decoder exceeds a threshold, the user is declared as a reliable symbol. The reliable information is fed back to the detection side and updates the symbol soft output when iterative processing is carried out. Numerical results show that system spectral efficiency is improved for 3L system over Rayleigh fading.

Index Terms - Nonbinary LDPC codes, Multicarrier MFSK, Multiple Access.
\end{abstract}

\section{Introduction}

For wireless communication systems, the increase of the number of users in a system is a growing demand to meet user's requirements. However, when the number of users increases, the multiple access interference (MAI) dominates the system performance. Therefore, multiuser detection (MUD) is an effective way to improve the system performance. For the hopping system, one of the most commonly known is the fast frequency hopping spread spectrum system with $M$-ary frequency shift keying (MFSK) [1], [2]. The system can mitigate multiple access interferences and fading effects. To increase system performance, several multiuser detection techniques have been proposed.A more detail review for these techniques can be found in [5].

In [6], [7], a multicarrier MFSK system was introduced with the features of frequency diversity and near far resistance. An IIC interference cancellation has been proposed [8]. The system performance is analyzed over Rician channel in [9]. In this paper, we study the soft multiuser detection for MC-MFSK MA system using powerful channel coding.

Low density parity check (LDPC) codes are one of the capacity approach error correction codes. These codes were first introduced by Gallager [10]. It can be efficiently decoded by using sum product algorithm (SPA) [11]. Nonbinary LDPC codes are similar to binary ones. It has been shown nonbinary LDPC codes outperform binary codes over binary AWGN channel [11]. Moreover, when use QSPA-FFT algorithm, the decoding complexity will reduced tremendously [11]. This motivates the applications of nonbinary LDPC codes in communication systems [13], [17], [18]. Furthermore, it avoid the conversion loss from symbol to bit conversion during message passing from detector to decoder. In this paper, we focus on nonbinary LDPC codes as the coding and decoding technique. Based on its binary LDPC codes, the nonbinary LDPC codes are constructed just replace each 1 in binary parity check matrix with nonzero elements random chosen from the corresponding Galois field. The binary parity check matrix can be constructed by progress edge growth (PEG) algorithm [16] to have the largest girth of the code. Note that the match between the order of the nonbinary code and the order of modulation alphabets will avoid symbol to bit conversion loss and vice versa.

Since the system under considered is a noncoherent system, the demodulation process is not a linear process. Hence, unlike coherent multiple access system, most multiuser detection algorithm of frequency hopping system remove the whole interfered signal [6]. We test the reliability of each user's symbol from decoder output. We count the number of reliable users on the received signals and compute the soft outputs from this information. As the iteration of detection and decoding proceeds, there will have more and more reliable detected users, and the system performance improved.

The rest of this paper is arranged as follows. Section 2 describes the system model. In Section 3, the multiuser soft output computation and an iterative detection and decoding algorithm are presented. In Section 4, simulation results are provided to show the system performances. Finally, conclusions are given in Section 5.

\section{System Model}

The system block diagram for user $k$ we consider is shown in Fig. 1. Assume that the number of orthogonal tones is $M$, and the number of bits per symbol is $b=\log _{2}(M)$. For each user, $b$ binary data bits are group in $b$-bit buffer, and mapped to an elements in $q$ order Galois Field $\operatorname{GF}\left(q=2^{b}\right)$. After encoded with a nonbinary LDPC code, the coded symbols are hopped with user's address to generate parallel transmit patterns. The parallel transmit patterns are then passed to an MFSK modulator to generate multiple orthogonal carriers. Let $L$ be the length of hopping address code. The address is an $L$-tuple unique pseudo random address $\mathbf{a}_{k}=\left(\mathrm{a}_{k, 0}, \mathrm{a}_{k},{ }_{1}, \ldots, \mathrm{a}_{k}, L-1\right)$, where $a_{k, l} \in G, \quad l=0,1, \ldots, L \quad$ and $G=\{0,1, \ldots, M-1\}$. The transmitted symbols $\mathbf{X}_{k}$ are generated by $\mathbf{X}_{k}=C_{m_{k}} \mathbf{a}_{k}$, where $C_{l}, C_{-l}$ represent shift to the top or shift to the bottom by 1 positions, respectively. As an example, let $m_{k}=2, \mathbf{a}_{k}=(1,3,7)$, then $\mathbf{X}_{k}=(3,5,1)$. The symbol rate is $R_{s}$ symbols/s, where $R_{s}=1 / T_{s}=1 /\left(b T_{b}\right)$. Hence, the MC-MFSK transmit $L$ parallel tones simultaneously within a symbol duration $T_{s}$ seconds and the system bandwidth is $W=M R_{s} \mathrm{~Hz}$. For coded 
multicarrier MFSK system, the spectrum efficiency is defined as $\eta=\log _{2}(M) \times U / M \times R_{c}$ bits $/ \mathrm{sec} / \mathrm{Hz}$, where $U$ is the total users in the system and $R_{c}$ is the code rate.

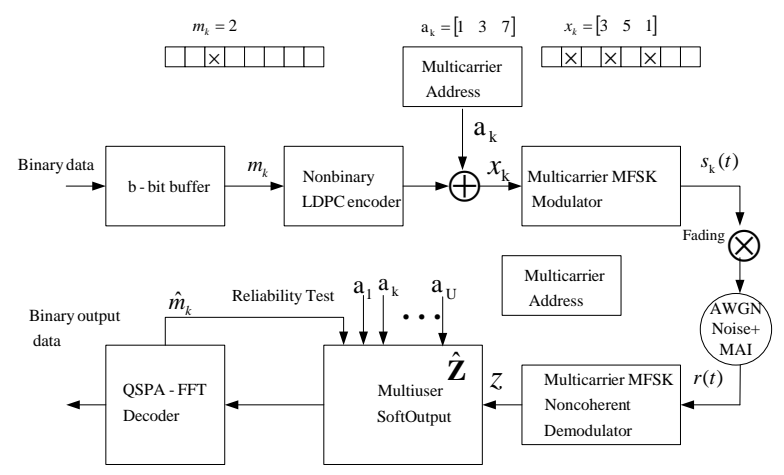

Fig. 1 System Block Diagram.

At the receiver, after multicarrier demodulation, signals are normalized with the average received energy. Without loss of generality, we assume user 1 as the desired user and we omit the user index in the following context. Assume there are $U$ total users and the system is a synchronous system. Then, the equivalent baseband complex signal on $n$th tone given the desired symbol is $m$ is given as

$$
r_{n}=\alpha_{m} \delta_{n m} e^{j \phi_{m}}+\sum_{j=1}^{U-1} \alpha_{j n} c_{j n} e^{i \phi_{j}}+w_{n}, \quad 0 \leq n \leq M-1
$$

where $\left\{\alpha_{m}\right\}$ and $\left\{\alpha_{j n}\right\}$ are independent, identical distributed (i.i.d.) random variables with $E\left[|\alpha|^{2}\right]=1$. Phases $\left\{\phi_{m}\right\}$ and $\left\{\phi_{j}\right\}$ are i.i.d. uniformly distributed random variables over $[0,2 \pi) .\left\{c_{j n}\right\}$ is 0 or 1 to indicate whether the $j$ th interferer user occupy the $n$th sub-channel. $\delta_{m n}$ is the Kronecker delta function with $\delta_{m n}=1$ if $m=n$, and zeros otherwise. $w_{n}$ denotes zero-mean complex Gaussian noise whose real and imaginary part have the same variance. The average symbol signal-to noise ratio (SNR) is $\gamma_{\mathrm{s}}=\bar{E}_{s} / N_{0}=\gamma_{b} \log _{2} M / L$, where $\gamma_{\mathrm{b}}$ is the bit energy-to noise ratio $\left(E_{b} / N_{0}\right)$, and $\bar{E}_{s}$ is average symbol energy .

\section{A. PDFs for Demodulated Signal over Rayleigh Fading \\ Channel}

In order to calculate soft metrics, it is necessary to know the probability density function of the received signal. Since the envelope detectors are used, the outputs of detectors, denoted as $z_{n}$ are $z_{n}=\left|r_{n}\right|$. These signals are stored in a buffer $\mathbf{Z}=\left[z_{0}, z_{1}, \ldots, z_{M-1}\right]^{T}$, where $\mathrm{T}$ is the transpose operator. The PDF of $z_{n}$ condition on the desired symbol $m$ over Rayleigh fading channel is given by

$$
\begin{aligned}
& f_{Z_{n}}\left(z_{n} \mid m\right)=\sum_{k=0}^{U-1} P(k ; U-1, \mu) \frac{2 z_{n}}{\delta_{m n}+k+1 / \gamma_{s}} . \\
& \exp \left(-\frac{z_{n}^{2}}{\delta_{m n}+k+1 / \gamma_{s}}\right)
\end{aligned}
$$

The term, $P(k ; U-1, \mu)$, is the probability of position $n$ of $\mathbf{Z}$ that is occupied by $k$ out of $U-1$ interferers and is given by

$$
P(k ; U-1, \mu)=\left(\begin{array}{c}
U-1 \\
k
\end{array}\right) \mu^{k}(1-\mu)^{U-1-k}
$$

where $\mu=\mathrm{L} / M$ is the independent, identical probability that each user occupy a tone.

Each element of buffer $\mathbf{Z}$ may be hit by multiple users. If by some ways, one can counts out how many reliable users occupy each element of buffer $\mathbf{Z}$, then we can modify the PDFs of the received signals more accurately. Let $\boldsymbol{\kappa}=\left[\kappa_{0}, \kappa_{1}, \ldots, \kappa_{M-1}\right]$ be the number of reliable users corresponding to $M$ positions of buffer $\mathbf{Z}$, where $\kappa_{i}$ represents the number of reliable users on the $i$ th position. Then, the PDF can be modified as

$$
\begin{aligned}
& f_{Z_{n}}\left(z_{n} \mid m, \kappa_{n}\right)= \\
& \sum_{k=0}^{U-1-\kappa_{n}} p\left(k ; U-1-k-\kappa_{n}, \mu\right) \times \frac{2 z_{n}}{\delta_{m n}+k+\kappa_{n}+1 / \gamma_{s}} \times \\
& \exp \left(-\frac{z_{n}^{2}}{\delta_{m n}+k+\kappa_{n}+1 / \gamma_{s}}\right)
\end{aligned}
$$

And, the CDF is given by

$$
\begin{aligned}
& F_{Z_{n}}\left(z_{n} \mid m, \kappa_{n}\right)=\sum_{k=0}^{U-1-\kappa_{n}} p\left(k ; U-1-k-\kappa_{n}, \mu\right) \times \\
& \left(1-\exp \left(-\frac{z_{n}^{2}}{\delta_{m n}+k+\kappa_{n}+1 / \gamma_{s}}\right)\right)
\end{aligned}
$$

\section{B. Hard-Limit Process}

To simplify the MUD process, the received signals are hard-limited to 2 levels $(2 L)$ with a proper threshold $\varepsilon_{0}$. The hard-limited buffer is denoted as $\hat{\mathbf{Z}}$. Each element, $\hat{z}_{n}, 0 \leq n \leq M-1$, of $\hat{\mathbf{Z}}$ thus are expressed as

$$
\hat{z}_{n}=\left\{\begin{array}{rr}
\text { '0' } & \text { noise only } \\
11 ' & \text { at least one user present }
\end{array}\right.
$$

Furthermore, the signal $z_{n}$ can also be hard-limited as threelevels $(3 L)$ as follows:

$$
\hat{z}_{n}=\left\{\begin{array}{rr}
\text { '0' } & \text { noise only } \\
\text { '1' } & \text { exact one user presents } \\
\text { '2' } & \text { at least two users present }
\end{array}\right.
$$

In this case, the second threshold $\varepsilon_{1}$ is determined by the intersection point of the PDF of exact one user and the PDF of at least two users.

\section{Soft Metrics of Multiuser Detection}

\section{A. A posteriori probability Symbol Soft Output}

Assume that each element $\hat{z}_{n}$ on $\hat{\mathbf{Z}}$ are independent, the transition probability of the received hard-limited buffer 
$\hat{\mathbf{Z}}$ condition on transmitted symbol $m$ and $\kappa_{n}$ reliable users occupy $n$th element $P\left(\hat{\mathbf{Z}} \mid m, \kappa_{n}\right)$ is given by

$$
P\left(\hat{\mathbf{Z}} \mid m, \kappa_{n}\right)=\prod_{n \in\left(\mathbf{a} C_{-n}\right)} P\left(\hat{z}_{n} \mid n, \kappa_{n}\right) \prod_{n \in(\mathbf{a C}-n)} P\left(\hat{z}_{n} \mid n, \kappa_{n}\right)
$$

where $\mathbf{a} C_{-n}$ and $\left(\overline{\mathbf{a} C_{-n}}\right)$ denote desired and undesired symbols that are cyclically shifted $n$ positions to the bottom of $\hat{\mathbf{Z}}$, respectively. Probability $P\left(\hat{z}_{n} \mid m, \kappa_{n}\right)$ is computed by equation (5). After normalization, we have,

$$
P(m \mid \hat{\mathbf{Z}}, \mathbf{\kappa})=\frac{P\left(\hat{\mathbf{Z}} \mid m, \kappa_{n}\right) P(m)}{\sum_{\tilde{m}=0}^{M-1} P(\hat{\mathbf{Z}} \mid \tilde{m})}
$$

The apriori probability $P(m)$ in equation (9) is set to $1 / M$ by the assumption that all symbols are transmitted equal likely. In addition, $\kappa$ is set to zero at the first time of the demodulation since there are no reliable information.

For the $2 L$ case, the probability of $P\left(\hat{z}_{n} \mid m, \mathbf{\kappa}\right)$ can be given as

$$
\begin{aligned}
& P\left(\hat{z}_{n} \mid m, \mathbf{\kappa}\right)= \\
& \left\{\begin{array}{l}
\sum_{k=0}^{U-1-\kappa_{n}} p\left(k ; U-1-k-\kappa_{n}, \mu\right) \times\left(1-\exp \left(-\frac{\varepsilon_{0}^{2}}{\delta_{m n}+k+\kappa_{n}+1 / \gamma_{s}}\right)\right) \text { if } \hat{z}_{n}=0^{\prime} \\
\sum_{k=0}^{U-1-\kappa_{n}} p\left(k ; U-1-k-\kappa_{n}, \mu\right) \times \exp \left(-\frac{\varepsilon_{0}^{2}}{\delta_{m n}+k+\kappa_{n}+1 / \gamma_{s}}\right) \quad \text { if } \hat{z}_{n}={ }^{\prime} \prime^{\prime}
\end{array}\right.
\end{aligned}
$$

And, for the $3 L$ case, the probability of $P\left(\hat{z}_{n} \mid m, \mathbf{\kappa}\right)$ is computed by:

$P\left(\hat{z}_{n} \mid m, \mathbf{\kappa}\right)=$

$$
\begin{cases}\sum_{k=0}^{U-1-\kappa_{n}} p\left(k ; U-1-k-\kappa_{n}, \mu\right) \times\left(1-\exp \left(-\frac{\varepsilon_{0}^{2}}{\delta_{m n}+k+\kappa_{n}+1 / \gamma_{s}}\right)\right) & \text { if } \hat{z}_{n}={ }^{\prime} 0 \\ \sum_{k=0}^{U-1-\kappa_{n}} p\left(k ; U-1-k-\kappa_{n}, \mu\right) \times \exp \left(-\frac{\varepsilon_{1}^{2}-\varepsilon_{0}^{2}}{\delta_{m n}+k+\kappa_{n}+1 / \gamma_{s}}\right) & \text { if } \hat{z}_{n}={ }^{\prime}{ }^{\prime} \\ \sum_{k=0}^{U-1-\kappa_{n}} p\left(k ; U-1-k-\kappa_{n}, \mu\right) \times \exp \left(-\frac{\varepsilon_{1}^{2}}{\delta_{m n}+k+\kappa_{n}+1 / \gamma_{s}}\right) & \text { if } \hat{z}_{n}=2^{\prime}\end{cases}
$$

As an example, Fig. 2 represents a hard-limited signals of buffer $\hat{\mathbf{Z}}$ with $M=8$. For each element of $\hat{\mathbf{Z}}$, the blank space represents hard-limited level ' 0 ' while ' 1 ' represent there are exactly one user and ' 2 ' represents at least two users in that position for $3 \mathrm{~L}$ system. Filled-pattern squares represent how many reliable users on that position. Assume the transmitted symbol $m=0$, then $\mathbf{a} C_{-n}=[0,2,4]$. Hence, the transition probability is given as

$$
\begin{aligned}
& P(\hat{\mathbf{Z}} \mid m, \mathbf{\kappa})= \\
& \left\{P\left(\hat{z}_{0}=1^{\prime} \mid n=0, \kappa_{1}=0\right) \cdot P\left(\hat{z}_{2}=I^{\prime} \mid n=2, \kappa_{2}=0\right) .\right. \\
& \underbrace{\left.P\left(\hat{z}_{4}=2^{\prime} \mid n=4, \kappa_{4}=4\right)\right\} \cdot}_{\text {desiredsymbols }} \\
& \left\{P\left(\hat{z}_{1}={ }^{\prime} 0^{\prime} \mid n=1, \kappa_{1}=2\right) \cdot P\left(\hat{z}_{3}=0^{\prime} \mid n=3, \kappa_{3}=3\right) .\right. \\
& P\left(\hat{z}_{5}=1^{\prime} \mid n=5, \kappa_{5}=0\right) \cdot P\left(\hat{z}_{6}=0^{\prime} \mid n=6, \kappa_{6}=1\right) . \\
& \underbrace{\left.P\left(\hat{z}_{7}=0^{\prime} \mid n=7, \kappa_{7}=0\right)\right\}}_{\text {undesiredsymbols }}
\end{aligned}
$$

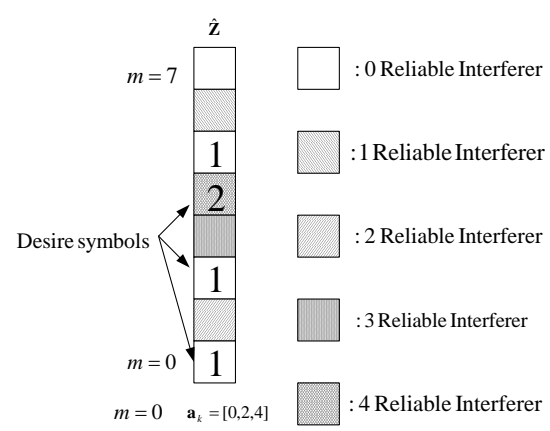

Fig. 2 Example for reliable users on $\hat{\mathbf{Z}}$ for $3 L$ sysem

\section{B. The proposed algorithm}

We pass the soft output from the multiuser detector to the channel decoding symbol-wise. We use QSPA-FFT algorithm for the decoding. If the reliability of a symbol exceeds a threshold, then this symbol is declared reliable, otherwise the symbol is unreliable.

Only unreliable symbols require further multiuser detection. We summarize the proposed iterative process as follows:

Step 1: Set those symbols whose soft outputs from channel decoder exceed a specified threshold as the reliable symbols.

Step 2: For those reliable symbols, regenerate the transmitted pattern by their own address code.

Step 3: Count the number of reliable users $\kappa_{i}, 0 \leq i \leq M-1$.

Step 4: Compute the soft outputs from $\hat{\mathbf{Z}}$, then pass the soft outputs to the LDPC decoder.

\section{Numeric Results}

When nonbinary channel coding is employed in $M$-ary communication system, the order of the Galois field of channel coding is chosen as the same as the size of the modulation alphabets. We choose the non-binary LDPC code over GF(128), GF(256) and GF(512) for 128-FSK, 256-FSK and 512-FSK MC systems, respectively. We use $(168,84)$ systematic regular non-binary LDPC code with symbol-node degree distribution $x$ [15]. The structure for the code is constructed by PEG algorithm. At the receiver, the detection threshold $\varepsilon_{0}$ for Rayleigh fading channels is set to $\varepsilon_{0}=3 / \sqrt{N_{0} / 2}$. The reliable testing threshold is obtained by try and error.

Since we need reliable users to carry multiuser detection, at the LDPC decoder, the maximum number of iterations in SPA algorithm is set to 15 for 128 -FSk and 5 for 256-FSK and 512-FSK, respectively. The maximum number of iteration between multiuser detection and channel decoder is set to 2 .

The frequency diversity order $L$ is another parameter which affects the system performance. Lower diversity $L$ will decrease the benefit of diversity gain, but higher diversity $L$ will increase multiple access interference. The value of $L$ is chosen from simulation. From the simulated SER of soft decision of the system, we can search the best $L$. In all the simulation cases, we set $L$ to 4 . 


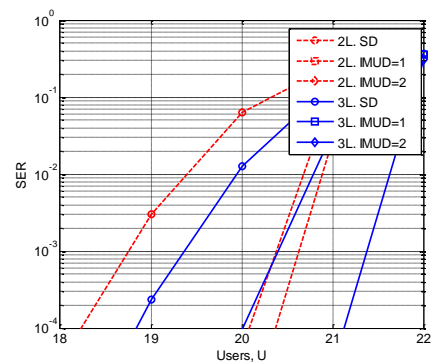

Fig. 3 Simulated BER vs. total users for a coded GF(128) transmission over Rayleigh channel; $R_{\mathrm{c}}=1 / 2 ; E_{\mathrm{b}} / N_{0}=25 \mathrm{~dB}$.

Fig. 3 shows the Performance of MC 128-FSK over Rayleigh fading with code rate $1 / 2$ and bit energy to noise ratio $E_{b} / N_{0}=25 d B$. SD denotes soft decision. IMUD is the number of iteration of multiuser detection and decoding. From the results, we can see that at symbol error rate $P_{s}=10^{-4}$, there are 18 users in the system. After 2 time's multiuser detection, system users increase to 20 , about $11 \%$ increasing. For $3 \mathrm{~L}$ system, there is one more user than $2 \mathrm{~L}$ system. The spectral efficiency for $2 \mathrm{~L}$ and $3 \mathrm{~L}$ system are $\eta=0.55$ and $\eta=0.57$, respectively.

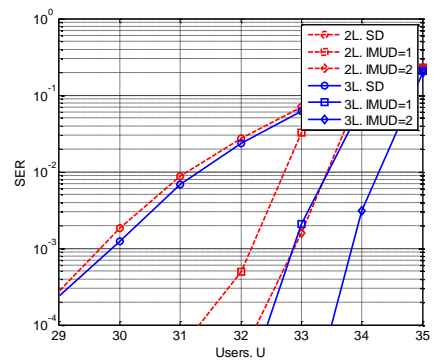

Fig. 4 Simulated BER vs. total users for a coded GF(256) transmission over Rayleigh channel; $R_{\mathrm{c}}=1 / 2 ; E_{\mathrm{b}} / N_{0}=25 \mathrm{~dB}$.

Fig. 4 is the performance of MC 256-FSK system over Rayleigh fading. At symbol error rate $P_{s}=10^{-4}$, the users for $2 \mathrm{~L}$ system is from 28 to 32 and from 28 to 33 for $3 \mathrm{~L}$ system. The spectral efficiency is $\eta=0.50$ and $\eta=0.52$, respectively.

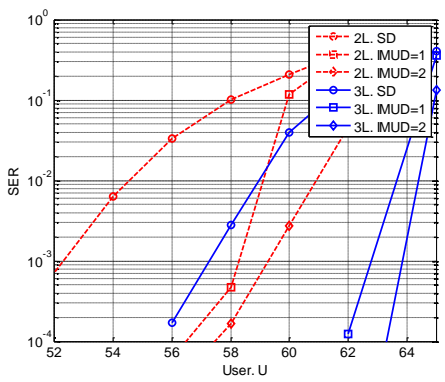

Fig. 5 Simulated BER vs. total users for a coded GF(512) transmission over Rayleigh channel; $R_{\mathrm{c}}=1 / 2 ; E_{\mathrm{b}} / N_{0}=25 \mathrm{~dB}$.

Fig. 5 shows the performance of MC 512-FSK systems over Rayleigh fading. At symbol error rate $P_{s}=10^{-4}$, the users for $2 \mathrm{~L}$ system is 57 and 63 for $3 \mathrm{~L}$ system. The spectral efficiency is $\eta=0.50$ and $\eta=0.55$, respectively.

\section{Conclusions}

In this paper, we have showed an iterative multiuser detection and decoding and showed that the application of nonbinary LDPC codes to MC MFSK multiple access systems have total users gains. When the soft metric at multiuser detection side is quantized to 3 levels can increase system performance than that of 2 levels system. We can see that nonbinary LDPC codes combined with symbol-wise receiver are very attractive for the systems considered.

\section{References}

[1] D. J. Goodman, P. S. Henry, and V. K. Prabhu, "Frequency-hopping multilevel FSK for mobile radio," Bell Syst. Tech. J., vol. 59, no. 7, pp.1257-1275, Sep. 1980.

[2] Yue, "Maximum Likelihood Combining for Noncoherent and Differentially Coherent Frequency-Hopping Multiple- Access Systems," IEEE Trans. on Infom. Theory, vol. IT-28, no. 4, pp. 631-639, 1982

[3] U. Fiebig, "Iterative Interference Cancellation for FFH/MFSK Systems," IEE Proceedings Communications, vol. 143 , No. 6, pp. 380-388, 1996.

[4] K. W. Halford and M. Brandt-Pearce, "Multi-stage Multi-user Detection for FHMA,"IEEE Trans. on Commun., vol. 48, no. 9, Sept. 2000, pp. 1550-1562.

[5] L.-L. Yang, Multicarrier Communications. Chichester, United Kingdom:John Wiley, 2009.

[6] R. Sinha and R. D.Yates, "An OFDM based multicarrierMFSK system,"in Proc. IEEE-VTS Fall VTC 2000, vol. 1, pp. 257-264.

[7] R. Sinha and R. D.Yates, "Performance of multicarrier MFSK in fading channels," in Proc.IEEE-VTS Fall VTC 2001, vol. 3, pp. 1848-1851.

[8] Zhan Yu, Tjeng Thiang Tjhung and Chin Choy Chai , "Multiuser Detection Algorithm Based On Iterative Interference Cancellation for MC-MFSK Systems," IEEE PIMR, , vol.4, pp. 2915-2919, 2004.

[9] Zhan Yu, Tjeng Thiang Tjhung and Chin Choy Chai , "Performance of Multiple Access Multicarrier MFSK System Over Rician Fading Channels," IEEE Trans. Veh. Technol., vol.54, pp. 1091-1102, May. 2005.

[10] R. G. Gallager, "Low-density parity-check codes," IRE Trans. Inform. Theory, vol. IT-8, pp. 21-28, Jan. 1968.

[11] D. J. C. MacKay, "Good error-correcting codes based on very sparse matrices," IEEE Trans. Inform. Theory, vol. 46, pp. 399-431, Mar. 1999.

[12] M. C. Davey and D. Mackay, "Low-density parity-check codes over GF(q)," IEEE Commun. Lett., vol. 2, pp. 165-167, June 1999.

[13] H. Song and J. R. Cruz, "Reduced-complexity decoding of Q-ary LDPC codes for magnetic recording," IEEE Trans. Magn., vol. 39, pp. 10811087, Mar. 2003.

[14] D. Declercq and M. Fossorier, "Decoding algorithms for nonbinary LDPCcodes over GF(q)," IEEE Trans. Commun., vol. 55, pp. 633-643, 2007.

[15] C. Poulliat, M. Fossorier, and D. Declercq, "Design of regular $(2, d c)-$ LDPC codes over $\mathrm{GF}(q)$ using their binary images," IEEE Trans.Commun., vol. 56, no. 10, pp. 1626-1635, Oct. 2008.

[16] Xiao-Yu Hu, E. Eleftheriou and D. M. Amold, "Regular and irregular progress edge-growth tanner graphs", IEEE Trans. Inform. Theory, vol. 51, no. 1, pp. 386-398, Jan. 2005.

[17] J. Huang, S. Zhou, and P. Willet, "Nonbinary LDPC coding for multicarrier underwater acoustic communication," IEEE J. Select. Areas Commun., vol.26, no. 9, pp. 1684-1696, 2008.

[18] S. Nowak and R. Kays,"High efficiency broadband transmission with LDPC codes over GF( $\left.2^{s}\right)$," 2011 IEEE International Symposium on Broadband Multimedia Systems and Broadcasting (BMSB) pp.1-6, June 2011. 